\title{
Noncollinear magnetic phases and edge states in graphene quantum Hall bars
}

\author{
J. L. Lado and J. Fernández-Rossier \\ International Iberian Nanotechnology Laboratory (INL), Av. Mestre José Veiga, 4715-330 Braga, Portugal
}

(Received 3 July 2014; revised manuscript received 1 October 2014; published 21 October 2014)

\begin{abstract}
Application of a perpendicular magnetic field to charge neutral graphene is expected to result in a variety of broken symmetry phases, including antiferromagnetic, canted, and ferromagnetic. All these phases open a gap in bulk but have very different edge states and noncollinear spin order, recently confirmed experimentally. Here we provide an integrated description of both edge and bulk for the various magnetic phases of graphene Hall bars making use of a noncollinear mean field Hubbard model. Our calculations show that, at the edges, the three types of magnetic order are either enhanced (zigzag) or suppressed (armchair). Interestingly, we find that preformed local moments in zigzag edges interact with the quantum spin Hall like edge states of the ferromagnetic phase and can induce backscattering.
\end{abstract}

DOI: 10.1103/PhysRevB.90.165429

PACS number(s): 73.43.Nq, 72.25.-b, 72.80.Vp, 75.76.+j

\section{INTRODUCTION}

The remarkably perfect [1] quantization of conductance in quantum Hall systems, that provides our standard of resistance for $h / e^{2}$, arises from the combined opening of a gap in the bulk of the sample, topologically different from vacuum, that implies the existence of chiral edge states for which backscattering is impossible [2,3]. In graphene, quantum Hall effect $[4,5]$ also shows perfect quantization, but it has its own peculiarities [6,7], as a result of the relativisticlike nature of the graphene electron dispersion, with two nonequivalent valleys with Dirac-like bands. In a honeycomb lattice, each valley hosts two sets of unevenly spaced Landau levels (LL), for electrons $n>0$ and holes $n<0$, plus a special $n=0 \mathrm{LL}$, at the Dirac energy. Whereas all graphene LL have fourfold degeneracy, coming from the spin and valley, the $n=0$ is different from the rest: for a given spin and a given edge, it has only one electronlike and holelike dispersive edge state.

It was found [8] early on that perturbations could open a gap in the $n=0$ LL in two fundamentally different ways, either by splitting the levels according to their spin, or to the valley (which is completely correlated to the sublattice for the $n=0 \mathrm{LL}$ ), resulting in very different edge states. Whereas breaking the sublattice symmetry would give gapped edge states [leftmost panel of Fig. 1(d)], the spin-polarized state would have counterpropagating edge states inside the gap [rightmost panel of Fig. 1(d)]. In this sense, the spectrum of spin-polarized graphene in the quantum Hall regime would be identical to the acclaimed quantized spin Hall phase proposed by Kane and Mele [9].

A generic feature of quantum Hall systems is that, when the Fermi energy lies at the middle of a Landau level, interactions can open a gap and break the spin symmetry. In the case of spindegenerate LL, this leads to the quantum Hall ferromagnetism. In the case of the $n=0$ graphene quartet, several types of electronic order have been studied [10-24]. Three obvious candidates come to mind: a charge density wave (CDW), a spin density wave resulting in antiferromagnetic (AF) order, both breaking valley symmetry, and ferromagnetic order (FM). Only the latter is expected to have gapless spin-filtered edge states [8,22].

Different experiments have provided strong evidence of interaction driven band gap opening in the $n=0$ quartet in graphene at half filling [25-27]. In a recent experimental breakthrough [28], the combined application of in-plane $B_{x}$ and off-plane $B_{z}$ fields has made it possible to observe the controlled transition between different electronically ordered phases at half filling. Thus, as $B_{x}$ is ramped up, the system goes from an insulating phase, most likely AF, to a phase with thermally activated edge transport, presumably a canted AF (CAF) phase with gapped edge states, and at higher in-plane field to a phase with $G$ slightly below $2 G_{0}$ (where $G_{0}=e^{2} / h$ ), as expected from the FM phase with quantum spin Hall like spectrum. Upon gating, all these phases merge into a phase with $G=G_{0}$.

These recent experimental results [28] highlight the interplay between noncollinear bulk electronic order and the emergence of spin-filtered edge states that, in contrast with the $n \neq 0$ states that are topologically protected, are the consequence of an interaction driven electronic phase transition in bulk. This motivates the interacting theory presented here, that describes on equal footing the noncollinear spin order of both bulk and edge states, going beyond previous theory work [10-24]. In particular, a previously overlooked but important aspect of this problem is the fact that the magnetic order is different at the edges and bulk. Our noncollinear mean field Hubbard model calculations show that zigzag and armchair edges enhance and suppress, respectively, the magnetic order associated to the bulk Landau levels. In the case of the recently observed quantum spin Hall like phase, the coexistence of spin filtered edge states with local magnetic moments at zigzag terminations is likely to play a role in the observation [28] of a conductance smaller than $2 G_{0}$ that is expected from these states in the absence of spin flip interactions [8].

The manuscript is organized as follows. In Sec. II we present the tight binding model and mean field approach used to model the quantum Hall bars. In Sec. III we present the results for the edge and bulk electronic properties at half filling, as well as for the $v=1$ phase. Finally, in Sec. IV we summarize our conclusions.

\section{MODEL}

We model graphene quantum Hall bars with a Hubbard model for a honeycomb lattice stripe:

$$
\mathcal{H}=\mathcal{H}_{0}\left(B_{z}\right)+g \mu_{B} \vec{B} \cdot \vec{S}+U \sum_{i} n_{i, \uparrow} n_{i, \downarrow} .
$$



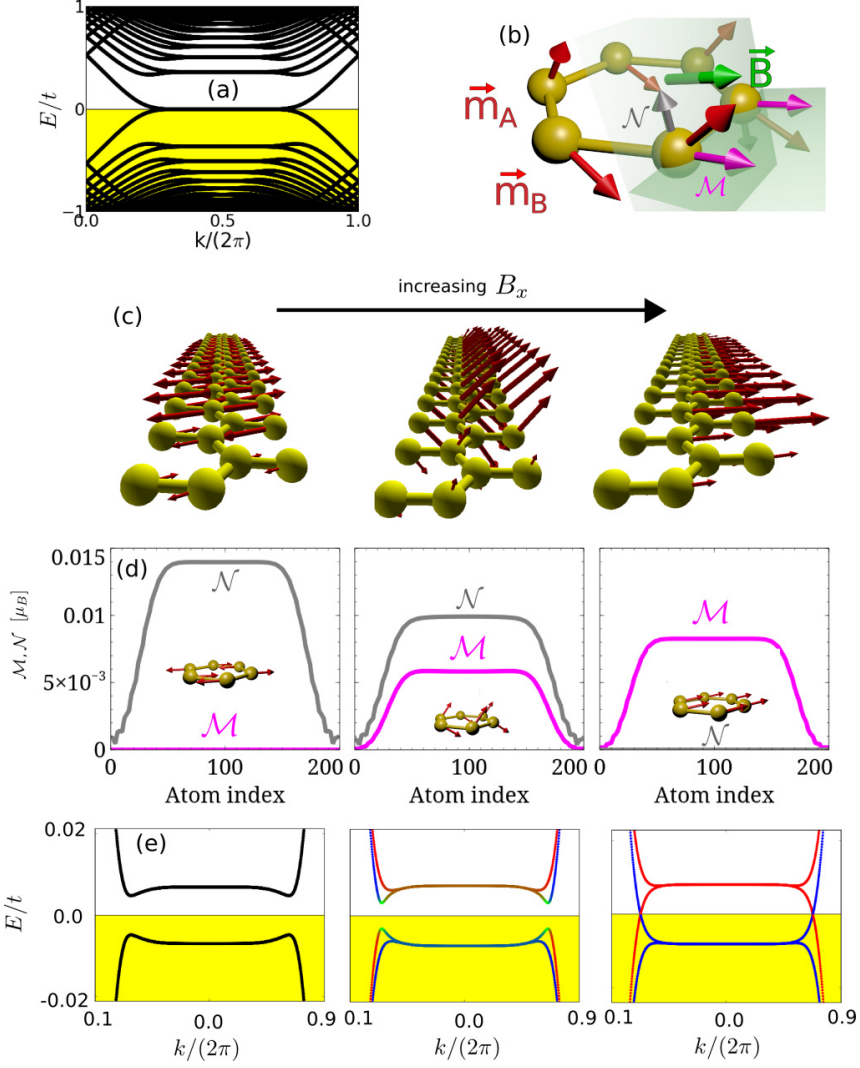

FIG. 1. (Color online) (a) Noninteracting spectrum of a quantum Hall armchair ribbon, and (b) scheme of the magnetism developed when interactions, off-plane, and in-plane fields are present. (c) Magnetism in the ribbon, (d) magnetic order parameters, and (e) band structures as the in-plane field increases, showing a pure edge insulator to metal transition.

The first term describes electrons in a honeycomb lattice and the effect of the perpendicular magnetic field $B_{z}$ on the orbital motion is included by means of the standard Peierls substitution in the tight binding model. The second term is the Zeeman coupling and the third is the Hubbard term, where $n_{i \sigma}=c_{i \sigma}^{\dagger} c_{i \sigma}$ is the occupation number for spin $\sigma$ at site $i$. Our gauge choice preserves translational invariance along the transport direction, so that $k$ is a good quantum number. The spectrum of $\mathcal{H}_{0}\left(B_{z}\right)$ is shown in Fig. 1(a) for a stripe with armchair terminations, and features both the bulk Landau levels and the dispersive edge states.

The effect of interactions is treated at the unrestricted Hartree-Fock approximation with a variational wave function $|\Omega\rangle=\prod_{i}\left(u_{i} c_{i \uparrow}^{\dagger}+v_{i} c_{i \downarrow}^{\dagger}\right)|0\rangle$. This naturally leads one to write the Hubbard part of the Hamiltonian as a one-body mean field Hamiltonian:

$$
H^{M F}=H_{0}+g \mu_{B} \vec{B} \cdot \vec{S}+H_{H}+H_{F}+E_{D C},
$$

where $H_{H}=U \sum_{\sigma} n_{i \sigma}\left\langle n_{i \bar{\sigma}}\right\rangle$ is the Hartree term, $H_{F}=-U \sum_{\sigma} c_{i \sigma}^{\dagger} c_{i \bar{\sigma}}\left\langle c_{i \bar{\sigma}}^{\dagger} c_{i \sigma}\right\rangle$ is the Fock term, $E_{D C}=-U\left[\left\langle n_{i \uparrow}\right\rangle\left\langle n_{i \downarrow}\right\rangle-\left\langle c_{i \uparrow}^{\dagger} c_{i \downarrow}\right\rangle\left\langle c_{i \downarrow}^{\dagger} c_{i \uparrow}\right\rangle\right] \quad$ is a constant, $\bar{\sigma}=-\sigma$, and $\langle O\rangle=\langle\Omega|O| \Omega\rangle$. The variational coefficients $v_{i}$ and $u_{i}$ are determined by iteration, starting from a trial solution, until a self-consistent solution is found. Solutions for bulk graphene, ignoring boundaries, can be found analytically [15] and are consistent with our numerical results. For strips, a numerical implementation of this procedure yields, in general, solutions with noncollinear magnetization whose magnitude and orientation vary from bulk to edge. Since a numerical calculation of the actual stripes, with one micron width, is beyond the reach of our computational resources, we consider narrower stripes with $W=10 \mathrm{~nm}$, with larger $B_{z}$, so that the magnetic length $\ell_{B}=\sqrt{\frac{\hbar}{e B_{z}}}$ that controls interedge coupling is still much smaller than $W$.

The magnetic order of a given self-consistent solution is completely characterized by the average spin moment in every atom of the ribbon unit cell, $\vec{m}_{i}=\left\langle\Omega\left|\vec{S}_{i}\right| \Omega\right\rangle$. It is convenient to introduce to two fields that measure the degree of ferromagnetic (FM) and antiferromagnetic (AF) order of a given solution. For each pair of adjacent atoms, $A$ and $B$, we define

$$
\vec{M}=\frac{\vec{m}_{A}+\vec{m}_{B}}{2}, \quad \vec{N}=\frac{\vec{m}_{A}-\vec{m}_{B}}{2}
$$

\section{RESULTS}

At half filling we find that $\left|\vec{m}_{A}\right|=\left|\vec{m}_{B}\right|$ which implies that $\vec{N}$ and $\vec{M}$ are orthogonal. We also find that $\vec{N}$ and $\vec{M}$ points always perpendicular and parallel, respectively, to the applied magnetic field, in order to minimize the Zeeman energy. The mean field Hamiltonian is invariant to rotations of $\vec{N}$ in the plane perpendicular to the applied field. Therefore, at half filling is enough to refer to $\mathcal{N}=|\vec{N}|$ and $\mathcal{M}=|\vec{M}|$.

\section{A. Bulk properties at half filling}

Both the evolution of the magnetic order parameters from edge to edge [Figs. 1(d) and 3(b)], as well as the band dispersion [Figs. 1(e) and 3(a)], make it clear that edges and bulk are very different. We first discuss the calculated properties of the bulk region, which are in line with previous theory work [15,22]. At half filling we find three different phases, depending on the value of $B_{x}$. In the limits $B_{x}=0$ and $B_{x} \rightarrow \infty$, the Hubbard interaction yields bulk in-plane antiferromagnetic order $(\mathcal{M}=0)$ and in-plane ferromagnetic order $(\mathcal{N}=0)$, respectively. As $B_{x}$ is ramped between these two extremes, both the $\mathcal{N}$ and $\mathcal{M}$ components survive, in the so-called canted AF (CAF) phase [15,22].

The three magnetically ordered phases, FM, AF, or CAF, open a bulk gap $\Delta$ in the $n=0$ quartet. In Figs. 2(b) and 2(c) we show how the magnitude of the bulk gap $\Delta$ remains initially constant as a function of $B_{x}$, whereas the $\mathcal{M}$ increases linearly and $\mathcal{N}$ is depleted according to a $\sqrt{1-\left(\frac{B_{x}}{B_{0}}\right)^{2}}$ law. Since $\mathcal{M}$ scales linearly with $B_{x}$ this result shows that $\Delta \propto \sqrt{\mathcal{M}^{2}+\lambda^{2} \mathcal{N}^{2}}$, and $B_{x}$ is actually driving a rotation of the $(\mathcal{N}, \mathcal{M}) \propto(\cos \theta, \lambda \sin \theta)$ vector [15]. Thus the FM, AF, and $\mathrm{CAF}$ phases can be interpreted as different realizations of a common multidimensional order parameter, rather than phases with different order parameters [15,22].

An important test for the model is the dependence of the bulk gap $\Delta$ on the off-plane magnetic field $B_{z}$. In the experiments, a roughly linear dependence [29] $\Delta \propto B_{z}$ was 
(a)
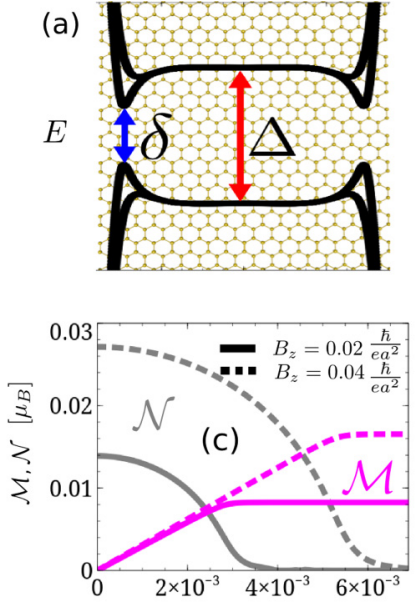

$B_{x}\left[t / \mu_{B}\right.$

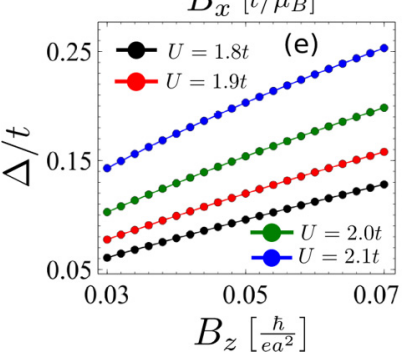

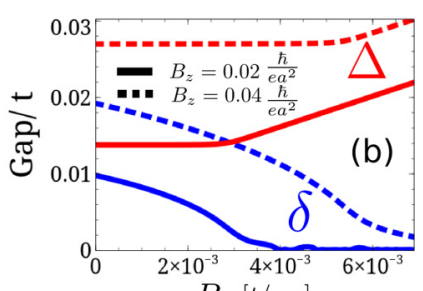

$B_{x}\left[t / \mu_{B}\right]$
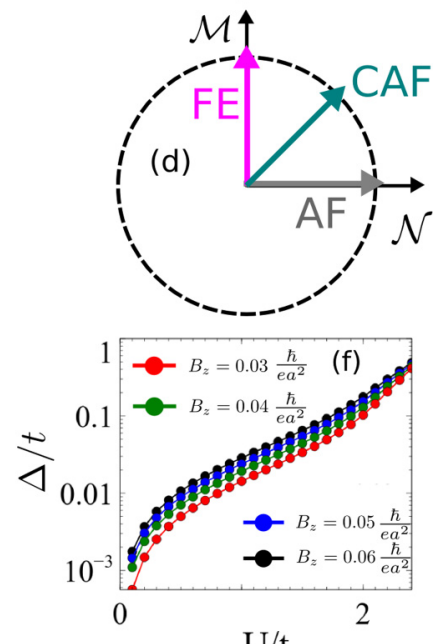

$\mathrm{U} / \mathrm{t}$

FIG. 2. (Color online) (a) Scheme of the two different gaps (edge and bulk) observed in the band structure for an armchair ribbon, and (b) their evolution with an increasing in-plane field. (c) Bulk magnetic order parameters obtained in the calculation, and (d) scheme representing the phase transition in the order parameter space. (e) Dependence of the AF gap in absence of in-plane field in a quantum Hall armchair bar as a function of the off-plane field, and (f) the electron-electron interaction.

found, in contrast with the expected $[10,11]$ from the HF theory for long-range Coulomb interaction, in which $\Delta \simeq \frac{e^{2}}{\ell_{B}} \propto \sqrt{B}$.

Within the mean field Hubbard model, the origin of the linear scaling is the following. First, the gap scales linearly with the atomic magnetic moment. Secondly, the magnetic

moment scales linearly with the number of electrons in the zero Landau level, which are the ones that are unpaired. This number of electrons is given by the ratio of the area of sample and the square of the magnetic length $A_{\text {sample }} / l_{B}^{2}$. This ratio is proportional to $B_{z}$, yielding the linear scaling of the gap.

The magnitude of the mean field gap depends strongly on $U$. In order to account for the experimentally observed magnitude of $\Delta /\left(e B a^{2} / \hbar\right)=40 \mathrm{eV}$, we would need to assume $U / t \simeq$ $2-2.5$, within the limits considered in the literature [30]. However, in order to calculated $\Delta$ it is probably more realistic to assume a smaller value for $U$ and to include the effect of long range Coulomb interaction as well $[10,11]$.

\section{B. Edge properties at half filling}

We now discuss the electronic properties of the edges. We consider both zigzag and armchair terminations. In both cases $\mathcal{N}$ and $\mathcal{M}$ are modulated as the edge is approached, but in a different way: they are depleted at the armchair edges [Figs. 1(c) and 1(d)] and enhanced at the zigzag terminations [Fig. 3(b)]. We start the discussion with the evolution of the edge states as a function of $B_{x}$ for the simpler case of armchair edges. As $B_{x}$ is ramped up, $\mathcal{N}$ is depleted in bulk, so it does the edge gap, $\delta$ [Fig. 2(b)]. Thus, in the AF phase, with $\delta \simeq \Delta \gg k_{B} T$ edges are insulating, but in the CAF phase, when $\mathcal{N}$ and $\delta$ are close to zero, thermally activated edge transport is possible, as reported experimentally [28]. In the ferromagnetic phase, with $\mathcal{N}=0$, the edge gap closes, $\delta=0$, and our calculated spectrum is identical to that of a quantum spin Hall insulator [9], with a finite gap $\Delta$ in the bulk spectrum and spin-polarized counterpropagating gapless edge states.

The existence of the quantum spin Hall like spectrum in the FM phase is true both for zigzag and armchair terminations, confirming the prediction based in a model that ignored the modulation of the order parameter at the edge [8]. However, in the zigzag edges the enhancement of the magnetic moment at the edges has nontrivial and important consequences. Two types of edge states exist at the zigzag terminations in the quantum Hall regime: the topologically protected current carrying states, present in any quantum Hall bar $(\mathrm{QH})$, and
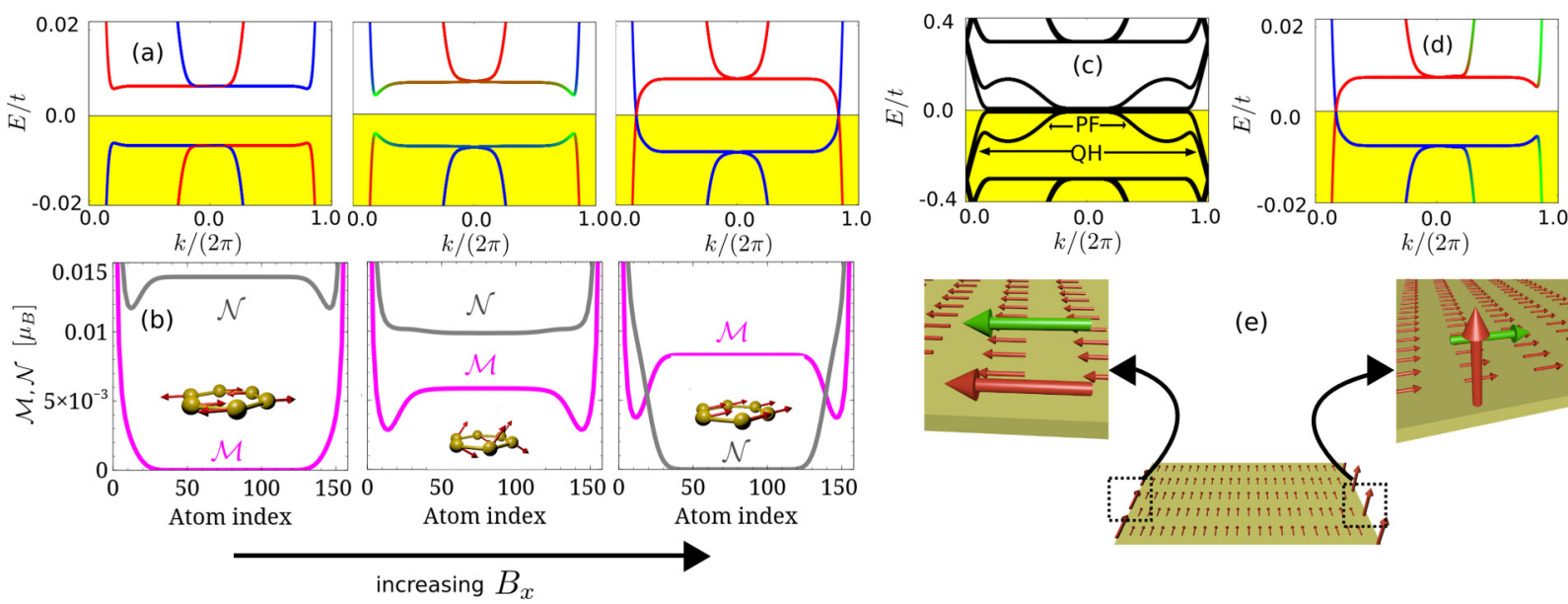

(e)

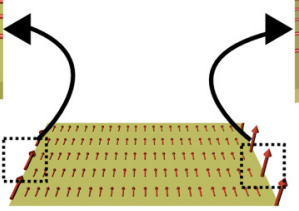

FIG. 3. (Color online) (a) Energy bands for the AF, CAF, and FM in a zigzag ribbon and (b) magnetic order parameters across the width. (c) Band structure of a zigzag ribbon marking the two different kinds of edge states. (d) Band structure and (e) scheme of a zigzag ribbon in the ferromagnetic regime with an excited magnetic moment on one edge. 
the preformed (PF) nondispersive midgap edge states [31], present already at $B_{z}=0$, that host magnetic moments when Hubbard interactions are turned on [32,33]. These two types of edge states, QH and PF, are marked in Fig. 3(c). Inspection of their wave function confirms that the PF edge states are mostly localized in the last atomic row of the stripe, in contrast with the $\mathrm{QH}$ states, that are extended over a distance of order $\ell_{B}$ [34]. The enhancement of the magnetic order at the zigzag edges comes from the interaction driven ferromagnetic order associated to the PF edge states. The calculations yield edge magnetic moments lying parallel to the applied magnetic field, i.e., mostly in-plane.

An important question is to which point the edge magnetic moments associated to the PF states are coupled to the $\mathrm{QH}$ edge states. To address this question we perform a mean field calculation for the FM phase (with $B_{x} \gg B_{z}$ ) where we constrain the magnetic moment of one of the edges to lie perpendicular to the plane [Fig. 3(e)]. The resulting self-consistent solution still has in-plane magnetization for bulk and for the free edge. The calculated energy bands are shown in Fig. 3(d). It is apparent that at the edge where the PF magnetic moments are forced to lie off plane, a gap opens in the $\mathrm{QH}$ edge states.

Our calculation clearly shows that spin-filtered QH edge states are sensitive to the spin orientation of the PF edge moments. This provides a natural scenario to account for the experimentally observed value [28] of the zero bias conductance $G=1.8 G_{0}$, rather than $G=2 G_{0}$, the conductance expected if no spin-flip interactions occur. In a micron size flake there will be several patches with zigzag terminations and PF edge states. There, spin fluctuations of the edge moments will induce spin mixing and backscattering of the spin-filtered $\mathrm{QH}$ edge states. A second mechanism that would induce spin backscattering combines spin-orbit coupling and disorder [35].

\section{Ferrimagnetic $v=1$ phase away from half filling}

As a final test for the model, we now discuss our results (Fig. 4) for the system away from half filling. Experiments [28] indicate that, upon gating up to quarter filling the three magnetic phases merge into a unique phase with edge conductance $G=G_{0}$ which means that both spin and valley degeneracy have to be broken. Our calculations show that at quarter filling [Figs. 4(a) and 4(b)], the system develops a ferrimagnetic phase [Fig. 4(c)]. Unlike the case of half filling, we have now $\left|\vec{m}_{A}\right| \neq\left|\vec{m}_{B}\right|$ and the magnetic moments of both sublattices are parallel to the total applied field. The different magnitude of the sublattice magnetizations $m_{A}$ and $m_{B}$, shown in Fig. 4(d), is a clear indication of the valley symmetry breaking.

The occupation of a unique LL, possible due to the valley and spin symmetry breaking, automatically implies that a single spin-polarized dispersive edge state accounts for the observation [28] of the $G=G_{0}$ plateau. Importantly, both the bulk magnetization and the number of edge channels in this ferrimagnetic phase are insensitive to the magnitude of the in
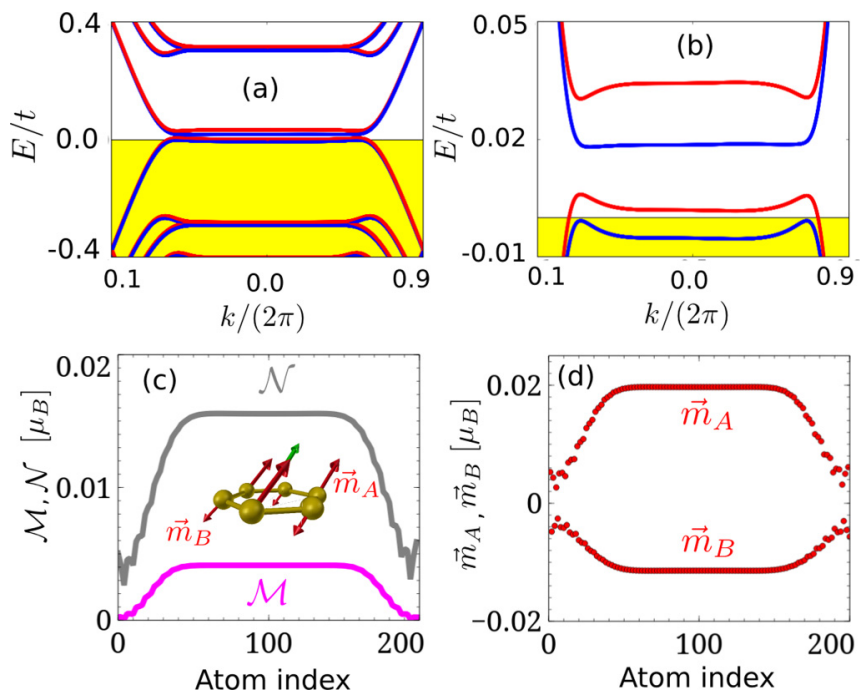

FIG. 4. (Color online) Quarter filling phase. (a) and (b) Energy bands, (c) magnetic order parameters, $\mathcal{N}$ and $\mathcal{M}$, as a function of position, and (d) sublattice resolved magnetic moments.

plane magnetic field, in agreement with the experiment [28]. Moreover, the bulk gap is found to increase with the in-plane field as observed in experiments.

\section{SUMMARY}

We have presented a comprehensive study of magnetic order in graphene stripes in the quantum Hall regime, mostly at half filling, based on a noncollinear mean field Hubbard model that treats both edge and bulk on equal footing. The interplay between bulk magnetism and the type of edge state, confirmed in recent experimental results [28], motivates the present work where the modulation of the magnetic order at the edges is taken into account. The model captures the main experimental observations including the linear scaling between the bulk gap $\Delta$ and the off-plane magnetic field $B_{z}$. Our calculations reveal the coupling between the quantum spin Hall like edge states and preformed local moments at zigzag edges, that provides a natural scenario for the spin back-scattering observed experimentally. This scenario is similar to recent proposals [36,37] where spin-Hall edge states interact with magnetic impurities. The last but not the least, at quarter filling the model predicts the existence of a previously overlooked ferrimagnetic phase with spin polarized edge states with $G=G_{0}$.

\section{ACKNOWLEDGMENTS}

We thank J. W. González for fruitful discussions. We acknowledge financial support by Marie-Curie-ITN 607904 SPINOGRAPH. J.F.R. acknowledges financial support by Generalitat Valenciana (ACOMP/2010/070), Framework Programme FP7/2007-2013/ under REA Grant Agreement No. 607904-13, Prometeo.
[1] K. Von Klitzing, Rev. Mod. Phys. 58, 519 (1986).

[2] R. B. Laughlin, Phys. Rev. B 23, 5632 (1981).
[3] B. I. Halperin, Phys. Rev. B 25, 2185 (1982).

[4] K. S. Novoselov et al., Nature (London) 438, 197 (2005). 
[5] Y. Zhang et al., Nature (London) 438, 201 (2005).

[6] M. I. Katsnelson, Graphene: Carbon in Two Dimensions (Cambridge University Press, Cambridge, UK, 2012).

[7] V. P. Gusynin and S. G. Sharapov, Phys. Rev. Lett. 95, 146801 (2005).

[8] D. A. Abanin, P. A. Lee, and L. S. Levitov, Phys. Rev. Lett. 96, 176803 (2006).

[9] C. L. Kane and E. J. Mele, Phys. Rev. Lett. 95, 226801 (2005).

[10] K. Nomura and A. H. MacDonald, Phys. Rev. Lett. 96, 256602 (2006).

[11] J. Alicea and M. P. A. Fisher, Phys. Rev. B 74, 075422 (2006).

[12] M. O. Goerbig, R. Moessner, and B. Douçot, Phys. Rev. B 74, 161407(R) (2006).

[13] V. P. Gusynin, V. A. Miransky, S. G. Sharapov, and I. A. Shovkovy, Phys. Rev. B 74, 195429 (2006).

[14] H. A. Fertig and L. Brey, Phys. Rev. Lett. 97, 116805 (2006).

[15] I. F. Herbut, Phys. Rev. B 76, 085432 (2007).

[16] J.-N. Fuchs and P. Lederer, Phys. Rev. Lett. 98, 016803 (2007).

[17] J. Alicea and M. P. Fisher, Solid State Commun. 143, 504 (2007).

[18] L. Sheng, D. N. Sheng, F. D. M. Haldane, and L. Balents, Phys. Rev. Lett. 99, 196802 (2007).

[19] J. Jung and A. H. MacDonald, Phys. Rev. B 80, 235417 (2009).

[20] A. A. Shylau and I. V. Zozoulenko, Phys. Rev. B 84, 075407 (2011).

[21] V. N. Kotov, B. Uchoa, V. M. Pereira, F. Guinea, and A. H. Castro Neto, Rev. Mod. Phys. 84, 1067 (2012).

[22] M. Kharitonov, Phys. Rev. B 85, 155439 (2012).

[23] M. Kharitonov, Phys. Rev. B 86, 075450 (2012).
[24] B. Roy, M. P. Kennett, and S. Das Sarma, arXiv:1406.5184.

[25] Y. Zhang, Z. Jiang, J. P. Small, M. S. Purewal, Y.-W. Tan, M. Fazlollahi, J. D. Chudow, J. A. Jaszczak, H. L. Stormer, and P. Kim, Phys. Rev. Lett. 96, 136806 (2006).

[26] Z. Jiang, Y. Zhang, H. L. Stormer, and P. Kim, Phys. Rev. Lett. 99, 106802 (2007).

[27] Y. J. Song, A. F. Otte, Y. Kuk, Y. Hy, D. B. Torrance, P. N. First, W. A. de Heer, H. Min, S. Adam, M. D. Stiles, A. H. MacDonald, and J. A. Strocio, Nature (London) 467, 185 (2010).

[28] A. F. Young, J. D. Sanchez-Yamagishi, B. Hunt, S. H. Choi, K. Watanabe, T. Taniguchi, R. C. Ashoori, and P. Jarillo-Herrero, Nature (London) 505, 528 (2014).

[29] C. R. Young, A. F. Dean, L. Wang, H. Ren, P. Cadden-Zimansky, K. Watanabe, T. Taniguchi, J. Hone, K. L. Shepard, and P. Kim, Nat. Phys. 8, 550 (2012).

[30] T. O. Wehling, E. Şaşığlu, C. Friedrich, A. I. Lichtenstein, M. I. Katsnelson, and S. Blügel, Phys. Rev. Lett. 106, 236805 (2011).

[31] K. Nakada, M. Fujita, G. Dresselhaus, and M. S. Dresselhaus, Phys. Rev. B 54, 17954 (1996).

[32] M. Fujita, K. Wakabayashi, K. Nakada, and K. Kusakabe, J. Phys. Soc. Jpn. 65, 1920 (1996).

[33] J. Fernández-Rossier, Phys. Rev. B 77, 075430 (2008).

[34] L. Brey and H. A. Fertig, Phys. Rev. B 73, 195408 (2006).

[35] J. W. González, J. L. Lado, and J. Fernández-Rossier (unpublished).

[36] A. M. Lunde and G. Platero, Phys. Rev. B 86, 035112 (2012).

[37] B. L. Altshuler, I. L. Aleiner, and V. I. Yudson, Phys. Rev. Lett. 111, 086401 (2013). 